\title{
La performance de l'administration et la gestion axée sur les résultats : il en faudra bien plus pour que la confiance des citoyens dans les institutions s'améliore !
}

\author{
Daniel Maltais, Professeur \\ École nationale d'administration publique
}

\section{INTRODUCTION}

$\mathrm{Au}$ cours de la dernière décennie, la gestion axée sur les résultats s'est imposée comme une approche incontournable pour qui s'intéresse à l'amélioration de la performance des administrations publiques. On peut associer cette approche au nouveau management public, un mouvement datant du début des années 80 qui a remis en question l'importance jugée trop grande du rôle des États démocratiques. Le nouveau management public a également remis en cause le mode bureaucratique prévalant dans les organisations publiques. Selon ce courant de pensée, le mode bureaucratique devrait-être enrayéa car il est inefficace. Sur ce point, plusieurs outils et concepts de gestion propre à l'entreprise privée (plan d'affaires, clients, livrables, etc.) ont été proposés sans pour autant avoir toujours amélioré la performance des organisations publiques. C'est dans cette perspective que ce texte propose une critique de la gestion axée sur les résultats, car elle ne semble pas avoir réussi à accroître le niveau de confiance des citoyens dans leurs administrations publiques. En effet, en tant que modèle d'optimisation de la performance des organisations publiques, la gestion axée sur les résultats se bute à des défis particuliers.

\section{LA PERFORMANCE ET LA GESTION AXÉE SUR LES RÉSULTATS}

Une des marques de commerce du nouveau management public est la nécessité pour les administrations et les organisations publiques d'améliorer leur performance. Ce terme peut prendre plusieurs sens et dans certains cas devenir le synonyme d'autres concepts dont ceux d'efficacité, d'efficience, de qualité de services, etc.

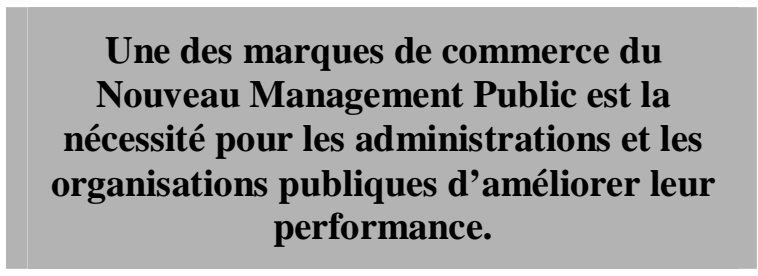

Deux définitions du terme «performance», tirées des dictionnaires, permettent de cerner les deux dimensions essentielles auxquelles il réfère. Pour le Grand Larousse Encyclopédique, «performance » est un : « mot anglais signifiant exécution, ... .achèvement. Par extension, exploit quelconque ». De son côté, l'Oxford English Dictionary définit le terme «performance» comme: «the accomplishment, execution, carrying out, working out of anything ordered or undertaken; the doing of any action or work; working, action ». Que l'on applique le terme à un individu qui pratique un sport ou encore à une organisation, la première définition met l'accent sur ce qu'on cherche à réaliser (un objectif, une vision) alors que la seconde attire l'attention sur les moyens ou les actions qui conduiront à cette réalisation (un entraînement spécifique, certaines techniques de gestion par exemple) ${ }^{\mathrm{b}}$.

Par conséquent, et selon ces deux définitions, parler de «performance », c'est réfléchir autant sur les résultats ultimes que l'on cherche à produire ou les objectifs que l'on cherche à atteindre $^{c}$ que sur les moyens utilisés pour y parvenir. 
Parler de « performance », c'est réfléchir autant sur les résultats ultimes que l'on cherche à produire ou les objectifs que l'on cherche à atteindre que sur les moyens utilisés pour y parvenir.

La « gestion axée sur les résultats », à la lumière de ces deux définitions, est étroitement associée à la notion de «performance ». Voulant réformer les bureaucraties publiques obsédées par l'application de règles tatillonnes et improductives, la gestion axée sur les résultats a principalement mis l'accent sur le volume et la qualité des services rendus par ces organisations ou encore sur les effets recherchés par leurs interventions. Pourtant, conceptuellement, la recherche de résultats peut tout autant être en lien avec l'atteinte d'objectifs liés à l'obtention de ressources (le recrutement de ressources humaines possédant une expertise particulière par exemple) ou encore en lien avec l'utilisation efficiente de ces ressources dans la production de services ou des effets obtenus.

En résumé, tout comme dans le cas de la performance, la gestion axée sur les résultats peut tout autant s'intéresser aux finalités poursuivies qu'aux moyens utilisés pour les réaliser. Parmi les auteurs qui ont prescrit une gestion axée sur les objectifs pour produire des résultats conséquents, Drucker ${ }^{1}$ compte certainement parmi les précurseurs.

Parmi les auteurs qui ont prescrit une gestion axée sur les objectifs pour produire des résultats conséquents, Drucker compte certainement parmi les précurseurs.
Au Québec, la Loi sur l'administration publique oblige les ministères et organismes gouvernementaux à adopter une gestion axée sur les résultats. Les ministères et organismes du gouvernement canadien sont aussi assujettis à cette discipline.

Les premières applications de cette approche dans les administrations peuvent aussi être retracées dans les années 60 quand le gouvernement fédéral américain introduisit, avec plus ou moins de succès, le Planning, Program, Budgeting System (PPBS). L'approche connaîtra un regain de popularité dans les années 80 dans les pays anglo-saxons, notamment en GrandeBretagne, en Australie, en Nouvelle-Zélande ainsi qu' au Canada. Chez certains universitaires, l'approche continue d'être promue. ${ }^{2}$

Ce regain de popularité ne signifie pas pour autant que les administrations produisent des résultats depuis peu, bien au contraire. Cependant, elles sont de plus en plus tenues de chercher à produire ceux qui sont en accord avec des objectifs préétablis et à questionner ceux qui ne sont pas conformes à ces derniers. Ainsi, au Québec, la Loi sur l'administration publique $^{3}$ oblige les ministères et organismes gouvernementaux à adopter une gestion axée sur les résultats. Les ministères et organismes $\mathrm{du}$ gouvernement canadien sont aussi assujettis à cette discipline. Bref, la plupart des administrations, dans leurs efforts de modernisation et d'amélioration de leurs performances, ont adopté la gestion axée sur les résultats.

\section{LA MODERNISATION DES ADMINISTRATIONS PUBLIQUES : PLUS QU'UNE AFFAIRE DE RÉSULTATS}

La performance et la gestion axée sur les résultats peuvent être discutées dans le cadre plus large des efforts que font la plupart des pays pour moderniser leur administration. Cette modernisation, qui peut être conçue comme une dynamique en continue, a pris des formes diverses au cours des ans et s'est installée à des rythmes variables selon les contextes des pays qui s'y sont engagés ${ }^{d}$. Tout en reconnaissant l'importance critique des contextes nationaux particuliers qui permettent au pays de s'inscrire plus ou moins rapidement et selon des modalités particulières, l'OCDE a identifié six leviers sur lesquels les pays développés se sont appuyés, 
plus particulièrement depuis une dizaine d'années, pour moderniser leur administration ${ }^{4}$.
Le tableau 1 présente ces leviers avec des exemples de pratiques auxquelles ils réfèrent.

Tableau 1 : Principaux leviers de modernisation des États

\begin{tabular}{|c|c|}
\hline Leviers & Exemples de bonnes pratiques $^{5}$ \\
\hline 1. L'administration ouverte & $\begin{array}{l}\text { Loi et mécanismes d'accès à l'information; services en lignes, services simplifiés; } \\
\text { consultations élargies auprès des citoyens. }\end{array}$ \\
\hline $\begin{array}{l}\text { 2. L'amélioration des performances du } \\
\text { secteur public }\end{array}$ & $\begin{array}{l}\text { Gestion et budgétisation axées sur les résultats; plus d'informations publiques sur } \\
\text { les performances. }\end{array}$ \\
\hline $\begin{array}{l}\text { 3. La modernisation de la } \\
\text { responsabilité et du contrôle } \\
\end{array}$ & $\begin{array}{l}\text { Plus d'imputabilité et de reddition de comptes; plus de contrôles a posteriori et qui } \\
\text { ne portent pas seulement sur les aspects financiers. }\end{array}$ \\
\hline $\begin{array}{l}\text { 4. La réaffectation des ressources et la } \\
\text { restructuration }\end{array}$ & Le réexamen de programmes entraîne des réorganisations... prudentes, agences. \\
\hline $\begin{array}{l}\text { 5. L'emploi des mécanismes de marché } \\
\text { dans la prestation des services } \\
\text { publics }\end{array}$ & Impartition / sous-traitance; partenariats public-privé; chèques services. \\
\hline $\begin{array}{l}\text { 6. L'organisation et la motivation des } \\
\text { fonctionnaires : la modernisation de } \\
\text { l'emploi public }\end{array}$ & $\begin{array}{l}\text { Attraction et rétention d'une main-d'œuvre jeune aux attentes élevées; la variété } \\
\text { des conditions offertes compte de plus en plus; et une gestion plus participative est } \\
\text { encouragée. }\end{array}$ \\
\hline
\end{tabular}

Cette liste de leviers montre que la gestion axée sur les résultats ou sur les performances ${ }^{f}$ constitue l'un des principaux leviers pour moderniser les administrations (levier \#2). Par ailleurs, on notera que plusieurs des six leviers traitent de moyens pour améliorer ces performances ou résultats ultimes: les services en ligne améliorent l'accessibilité des services publics (levier \#1), la création d'agences vise à enrayer la bureaucratie nuisible à la prestation de ces services (levier \#4), la sous-traitance permet d'utiliser les services d'organismes privés (levier \#5) et une gestion plus participative cherche à tirer le maximum des capacités des employés de l'État (levier \#6).

\section{La modernisation est une dynamique complexe et multiforme et l'amélioration des performances des États peut se compliquer dès lors qu'il faille en parallèle traiter d'autres dimensions.}

Cette multiplicité de leviers permet de mettre en relief un premier élément caractéristique de la gestion de la performance ou de la gestion axée sur les résultats dans le secteur public: la modernisation est une dynamique complexe et multiforme et l'amélioration des performances des États peut se compliquer dès lors qu'il faille en parallèle traiter d'autres dimensions. Ce qu' on attend des organisations publiques ne se limite donc pas à des résultats, concrets et mesurables, bien que l'atteinte de résultats en lien avec des objectifs convenus ait pris de plus en plus d'importance au cours des dernières décennies. On demande aussi de porter une attention toute particulière à une plus grande ouverture et une plus grande transparence des États (levier \#1), à plus de responsabilisation et à un meilleur contrôle (levier \#3), à l'application des lois, à la gestion des ressources et de l'information, l'ensemble de ces exigences ne concourant pas toujours à la production optimale de résultats ${ }^{\mathrm{g}}$.

À leur façon, plusieurs auteurs ont souligné cette complexité et la prudence avec laquelle on doit introduire des outils de modernisation comme la gestion axée sur les résultats. Kernaghan et al. ${ }^{5}$, par exemple, dans leur présentation d'un modèle post-bureaucratique, rappellent les distinctions importantes entre les organisations publiques dont la mission est d'élaborer et de formuler les grandes orientations de l'État, de produire des avis et des recommandations, celles dont le rôle est de faire respecter des lois ou règlements, d'émettre des permis et d'en faire respecter les conditions d'obtention et celles qui assurent la prestation de services publics auprès de la population. Dans le cas de ces dernières, on peut plus aisément appliquer la gestion axée sur les résultats, mais que dire des autres types d'organisations? 
Comment, par exemple, soumettre un ministère des finances à l'application stricte d'une gestion axée sur les résultats dans un contexte d'incertitude économique? Ou encore, quelle est la pertinence de considérer comme des « clients » des citoyens qui ont failli à leurs obligations et qui, dans le pire des cas, sont incarcérés?

\section{LA CONFIANCE : LA DIMENSION NÉGLIGÉE DANS LA RÉFLEXION DE LA PERFORMANCE DES ADMINISTRATIONS}

Le cadre analytique de Bouckaert $^{6}$ (figure 1), associe la performance dans le secteur public à un processus systémique de transformation d'intrants (ressources) en extrants (produits ou services) produisant des effets dont on espère qu'ils sont conformes aux objectifs poursuivis, lesquels devraient avoir été établis à partir d'une définition de besoins. Les relations entre les éléments de ce processus donnent lieu à des critères de performance bien connus dont l'économie, l'efficience et l'efficacité. Comme nous l'avons signalé plus tôt, la gestion axée sur les résultats pourra viser à améliorer la performance de l'organisation sur l'un ou l'autre de ces critères (et non seulement de l'unique point de vue de l'efficacité).

Figure 1 : Cadre analytique de la performance dans le secteur public

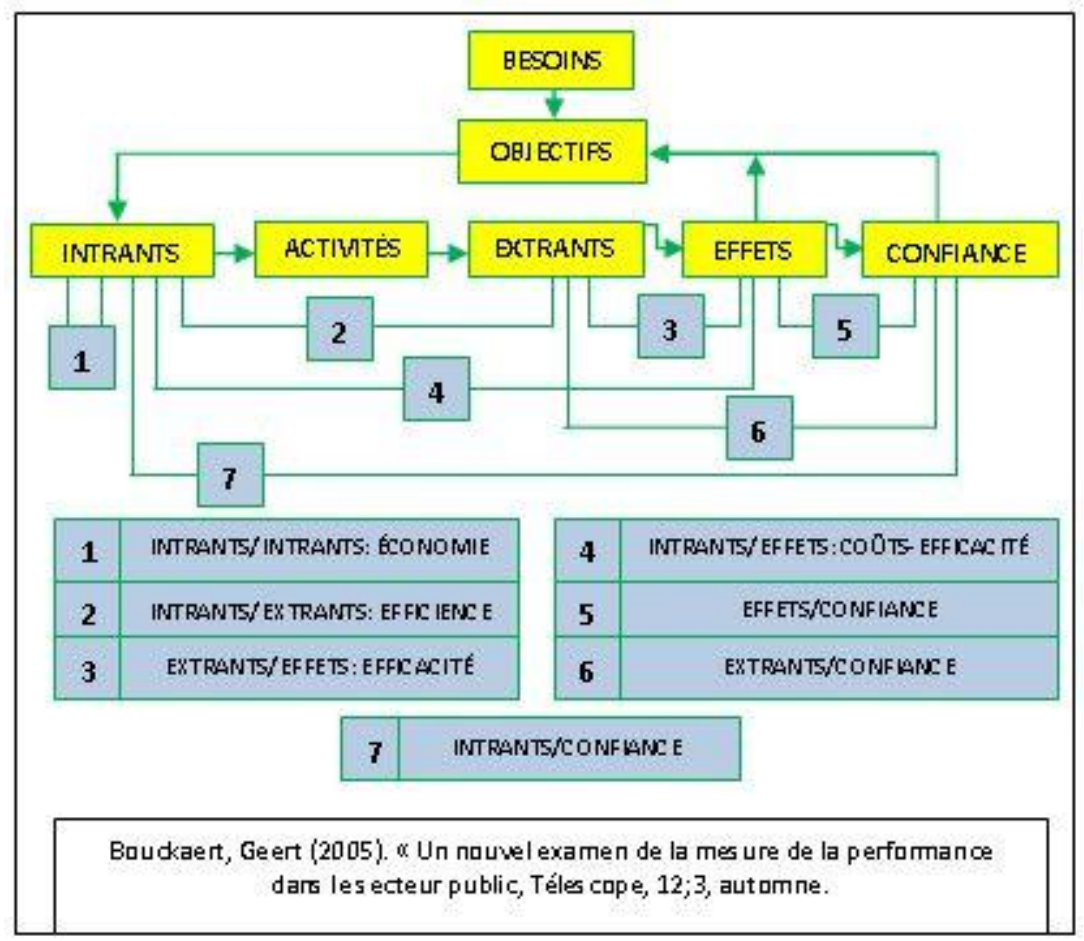

Un élément critique, que l'auteur met en relief dans son modèle, mais que nous jugeons avoir été insuffisamment traité voire négligé en matière de gestion de la performance ou de la gestion axée sur les résultats dans les organisations publiques, est la confiance des citoyens dans leurs institutions et leurs administrations.
Quelques événements récents illustrent l'importance de cet élément. Ainsi, à l'automne 2008, il est devenu évident que les États devraient faire face à une crise financière exceptionnelle (explosion de la bulle immobilière américaine, faillite de banques américaines, etc.) dont les effets sur l'économie réelle à l'échelle 
mondiale ne tarderaient pas à se faire sentir (faillites, pertes d'emplois, etc.). Les pressions de toutes provenances se sont alors manifestées pour que les États annoncent des mesures visant à résoudre cette crise sinon à en atténuer les effets. Le gouvernement canadien n'a pas eu d'autres choix que d'intervenir massivement en lançant rapidement un programme d'infrastructures et en fournissant des garanties financières à certains secteurs économiques en crise, dont celui de l'automobile. Quels objectifs concrets poursuivait-on? Protéger des emplois, en créer pour ceux qui perdraient le leur, soutenir les exportations canadiennes (surtout celles qui sont destinées à notre principal client, les ÉtatsUnis)? Des objectifs qui répondent certes aux attentes de la population en général, mais des objectifs imprécis qu'il sera difficile de mesurer à partir des résultats obtenus. Plusieurs douteront que les interventions gouvernementales aient été vraiment responsables des effets produits. Bref, qu'on doute ou pas de la capacité d'un gouvernement de s'inscrire dans une dynamique de gestion axée sur les résultats dans de telles circonstances, il appert que la performance du gouvernement est d'abord évaluée sur la confiance que suscitent dans la population les mesures annoncées, les milliards de dollars investis, autant d'intrants dont on ne sait pas dans quelle mesure ils seront déterminants et responsables de la sortie de crise.

Un autre exemple tient aux mesures gouvernementales visant à «faire face » à la pandémie de grippe A-H1N1, dont on ne sait pas, au moment d'écrire ces lignes, quelle en sera la portée et la gravité. Dans ce cas tout comme dans le cas précédent, la performance du gouvernement réside dans sa capacité à rassurer la population sur les moyens mis en place pour faire face au problème. Pourtant, rien ne nous assure que ces mesures produiront les effets escomptés. Ce qui compte pour l'instant c'est la confiance que suscitent les mesures prises.

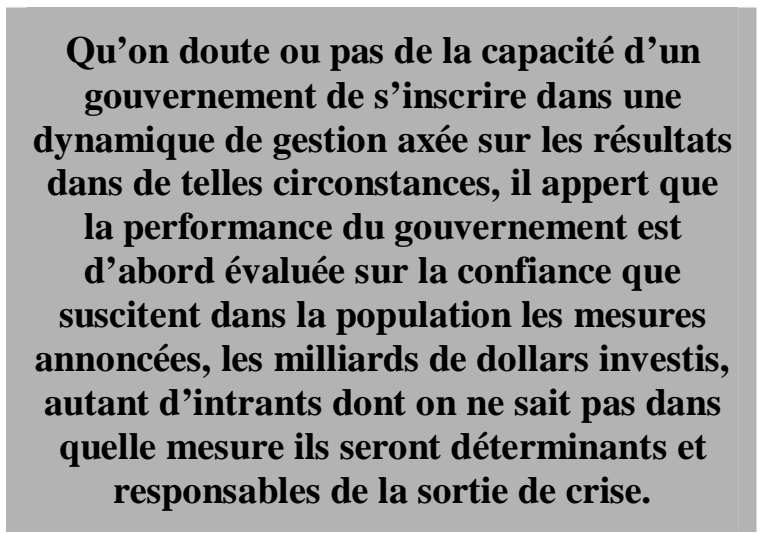

\section{UN PARADOXE : UNE CONFIANCE QUI S'EFFRITE MALGRÉ UNE GESTION DAVANTAGE AXÉE SUR LES RÉSULTATS}

La diminution de la confiance des citoyens dans leurs institutions démocratiques est une tendance lourde des dernières années. Qu'on mesure cette perte de confiance par la diminution de la participation aux élections, par la piètre opinion qu'on a des politiciens et des fonctionnaires ou encore par le cynisme à l'endroit des organisations publiques, il est pour le moins paradoxal que la gestion axée sur les résultats, maintenant en place depuis près d'une décennie, ne semble pas avoir enrayée la crise de confiance. Pourtant, jamais les élus et les citoyens n'ont eu autant d'informations sur leurs administrations. Ainsi, dans le cas du gouvernement du Québec, on peut retrouver sur les sites internet des ministères et organismes leurs plans stratégiques, leurs rapports annuels, des engagements fermes au regard des normes de services aux citoyens. L'Assemblée nationale, appuyée par le Vérificateur général, a même une commission parlementaire de l'administration publique qui est dédiée à l'examen de ces documents et à l'audition des responsables des ministères et organismes. Les citoyens seraient d'ailleurs étonnés de l'importance des effectifs et des budgets que les ministères consacrent à la production de données et de rapports alimentant les administrateurs et les ministres invités à rendre des comptes sur ce que font ou ne font pas leurs ministères. Alors comment expliquer que toute cette activité n'ait pas l'effet escompté sur la confiance des citoyens? Cette section propose quelques explications. 
Une première explication tient simplement à l'incapacité, pour les gouvernements, dans certaines circonstances et dans l'accomplissement de certaines missions, d'assurer la production de résultats en lien avec des objectifs préétablis et précis. Les deux exemples présentés plus tôt pourraient être repris ici. Ce type de situation est loin d'être exceptionnel : il est bien connu des professionnels devant agir sur des situations qu'ils ne contrôlent pas totalement ce qui les empêche de s'engager à obtenir des résultats. Un avocat n'est simplement pas en mesure de vous garantir les résultats d'une poursuite qu'il engage en votre nom et un chirurgien ne peut être absolument certain des résultats d'une opération délicate. Leur responsabilité tient à l'utilisation des moyens les plus pertinents dans les circonstances et non pas à l'assurance de produire des résultats par ailleurs souhaitables et recherchés, et ce, même si notre choix d'un professionnel repose en bonne partie sur ses succès passés en terme de résultats. L' "obligation de prendre les bons moyens », sans pour autant que ces moyens garantissent certains résultats, est une réalité insuffisamment expliquée aux citoyens par les tenants de la gestion axée sur les résultats et par nos politiciens.

\section{L' « obligation de prendre les bons}

moyens », sans pour autant que ces moyens garantissent certains résultats, est une réalité insuffisamment expliquée aux citoyens par les tenants de la gestion axée sur les résultats et par nos politiciens.

Une seconde explication tient à la perspective politique dans laquelle opère la gestion publique, en particulier dans une démocratie où un parti politique assume le pouvoir exécutif auquel doivent s'opposer d'autres partis politiques (partis d'opposition). Ceci signifie que, si on ne peut pas s'objecter dans ce type de régime aux principes d'imputabilité et de transparence au chapitre des décisions prises par les gouvernements, il nous faut accepter que l'application de ces principes s'inscrive dans un contexte partisan, non neutre, où l'information sur des résultats sert le plus souvent, soit à bien faire paraître, soit à mal faire paraître le gouvernement en place. On n'a ici qu'à évoquer les rapports du Vérificateur général dont on ne retient que ce qui peut alimenter la controverse politique. Pourtant, les lire montre que leur contenu est beaucoup plus nuancé que les histoires d'horreur qu'on en tire et qui font les manchettes. La crise qu'a connue le ministère canadien des ressources humaines ${ }^{\mathrm{h}}$ au début des années 2000 est un bel exemple qui montre bien comment la médiatisation d'un problème relativement mineur peut se transformer en crise majeure dans notre système politique. ${ }^{7}$

\section{Une troisième explication tient au culte de la quantification auquel donne lieu la gestion axée sur les résultats et qu'expose très bien Paquet. En bref, ce culte prescrit que tout ce qui ne peut pas être quantifié, à la limite... ne compte pas!}

Une troisième explication tient au culte de la quantification auquel donne lieu la gestion axée sur les résultats et qu'expose très bien Paquet. ${ }^{8}$ En bref, ce culte prescrit que tout ce qui ne peut pas être quantifié, à la limite... ne compte pas! Ce dogme a donné lieu à quelques phénomènes particulièrement inquiétants. Premièrement, ce sont les résultats qu'on peut plus facilement comptabiliser et produire à court terme qui deviennent prioritaires; les objectifs à long terme et plus difficilement quantifiables sont souvent négligés même si, à l'analyse, ils sont plus importants. Un deuxième phénomène tient à un investissement, démesuré dirons-nous, pour identifier des indicateurs, concevoir des systèmes de collecte et de traitement d'informations qui monopolisent une part importante des ressources des organisations publiques à tel point qu'on assiste à une nouvelle forme de bureaucratie pour qui tout doit être chiffré (pour l'ancienne, tout devait être réglementé). Encore une fois, si ce culte de la quantification contribuait à assurer une meilleure reddition de comptes de la part de nos élus et de nos gestionnaires publics, ces dérives pourraient être présentées comme des dommages collatéraux de la gestion axée sur les résultats. Pourtant, selon l'OCDE ${ }^{4}$, les responsables politiques n'utiliseraient que relativement peu tous ces chiffres: "Dans $72 \%$ des pays membres de l'OCDE, les objectifs sont systématiquement présentés dans la documentation 
budgétaire soumise au Parlement. Toutefois, dans seulement $19 \%$ des pays, les parlementaires utilisent les évaluations de performance dans la prise de décision. »

\section{L'imputabilité de nos dirigeants et des gestionnaires publics ne semble pas s'être améliorée malgré tous ces rapports chiffrés sur les résultats concernant les programmes ou les services publics.}

De plus, selon Savoie ${ }^{9}$, l'imputabilité de nos dirigeants et des gestionnaires publics ne semble pas s'être améliorée malgré tous ces rapports chiffrés sur les résultats concernant les programmes ou les services publics. Dans les faits, la gestion axée sur les résultats n'aurait que marginalement amélioré l'imputabilité de nos dirigeants politiques ou administratifs pour plusieurs causes. Parmi elles, notons :

ñ la persistance des ambiguïtés sur le plan de l'imputabilité politique et administrative;

ñ la concentration du pouvoir politique dans les mains d'un premier ministre et de son cercle restreint qui cherchent à tout contrôler;

ñ l'absence de ressources permettant aux élus d'analyser le formidable volume d'informations produit par les ministères et les organismes;

ñ le faible niveau du capital politique qu'un élu tirerait de l'analyse du volume excessif d'informations;

ñ la tendance à diluer les responsabilités et faire porter le chapeau à d'autres;

ñ les manœuvres bureaucratiques.

Radin ${ }^{10}$ croit que cette école de pensée axée sur la performance des organisations publiques a tout simplement failli. Elle fonde ce jugement péremptoire sur l'analyse d'une série de cas où l'application des prescriptions de ce mouvement n'a pas été possible. Cette faillite n'est pas due à des problèmes techniques mais aux fondements mêmes sur lesquels s'appuie ce mouvement, fondements qui ne valent pas pour les organisations publiques. La faillite est imputable au manque de réalisme des fondements de ce courant théorique qui postule de façon simpliste que : ñ la compréhension du monde peut s'appuyer sur des principes universels et clairs;

ñ le monde est linéaire; il est aussi une continuation du passé;

ñ les théories en gestion et les principes qui en émanent s'appliquent à tout type d'organisation;

ñ l'exercice des professions au sein des bureaucraties doit être étroitement contrôlé;

ñ l'obligation de valoriser les valeurs d'efficience et de rationalité dans les décisions;

ñ les représentants politiques peuvent s'accorder sur les grands objectifs et stratégies à poursuivre;

ñ l'information nécessaire est disponible, neutre et permet d'établir des liens de causalité entre les objectifs poursuivis et les moyens utilisés.

Ainsi, le nouveau management public, un courant de pensée axé sur la performance et la quantification excessive, repose sur de nombreuses hypothèses restrictives et peu représentatives des réalités organisationnelles publiques. Pour saisir le fonctionnement des organisations publiques et mieux comprendre leurs performances, un regard alternatif s'impose car des postulats bien différents caractérisent les systèmes publics. Ces postulats alternatifs renvoient à :

ñ une compréhension partielle du monde laquelle donne lieu, par ailleurs, à des interprétations multiples par divers groupes d'intérêt;

$\tilde{n}$ une poursuite par les organisations publiques d'objectifs nombreux et souvent conflictuels (l'équité et l'efficience par exemple);

ñ une nécessité de reconnaître et d'accorder l'autonomie nécessaire aux professionnels qui y œuvrent malgré le risque d'erreurs;

ñ la motivation élevée des représentants politiques d'être d'abord élus ou réélus;

ñ une information pas toujours disponible, coûteuse et souvent biaisée.

Le nouveau management public, un courant de pensée axé sur la performance et la quantification excessive, repose sur de nombreuses hypothèses restrictives et peu représentatives des réalités organisationnelles publiques. 


\section{CONCLUSION}

Si la gestion de la performance et la gestion axée sur les résultats ont pu et peuvent encore contribuer à améliorer le fonctionnement de nos administrations, la thèse que nous avançons dans cet article est que leur application dans le secteur public doit tenir compte des limites que lui impose notre contexte politique et institutionnel. De plus, nous sommes d'avis que ces approches n'ont pas contribué à accroître la confiance des citoyens dans leurs administrations, ce qui est pour le moins paradoxal. Enfin, si un des objectifs étaient de contrer la bureaucratie tant dénoncée, on peut douter de leur succès à ce sujet. Pire, la gestion de la performance et la gestion axée sur les résultats auraient contribué au développement d'un autre type de bureaucratie, surproductrice de données et de chiffres aux interprétations multiples et divergentes.

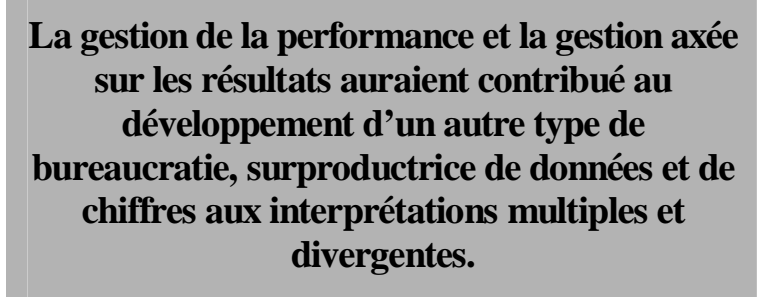

Sur le sujet de la bureaucratie tant décriée, il est utile de rappeler que certains croient qu'elle demeure, malgré tout, l'outil indispensable d'un État moderne. C'est notamment le point de vue de Suleiman ${ }^{11}$ en soulignant que : "Affaiblir la bureaucratie, que ce soit par le transfert de ses fonctions à des organismes privés, par la simple abolition de services ou par la réintroduction d'un système quasi clientéliste, n'est pas une simple question de «rationalisation administrative » ou d' « efficacité administrative ». Lorsqu'on affecte la capacité de l'État à produire et/ou mettre en œuvre des décisions politiques, et à assurer les services d'une manière efficace et relativement équitable, la question de la bureaucratie devient alors, par la force des choses, une question de démocratie. » Wilson $^{12}$ va dans le même sens en notant que : «All complex organizations display bureaucratic problems of confusion, red tape, and the avoidance of responsibility. Those problems are much greater in government bureaucracies because government itself is the institutionalization of confusion ... of red tape ... and of avoided responsibility. In short, you can have less bureaucracy only if you have less government. » Ces points de vue et les résultats discutables qu'ont produits, à ce jour, les réformes de nos administrations soulèvent la question importante du type de réformes qu'il faudra mettre en place au cours des prochaines années. Quelles qu'elles soient, nous nous rallions au point de vue de l'OCDE $^{4}$ pour qui ces réformes devront porter sur la gouvernance, laquelle : « exige qu'une grande attention soit accordée à des valeurs fondamentales telles que la loyauté, l'équité, la justice et la cohésion sociale afin que soit préservée la confiance à l'égard du système gouvernemental et politique considéré dans son ensemble. Les questions de gestion, pour importantes qu'elles soient, doivent passer au second plan. »

\section{Bibliographie}

1 Drucker, P. F. (1964). Managing for Results, London : William Heinemann Ltd, 270 .

2 Mazouz, B. et J. Leclerc (2008). Gestion intégrée par résultats. Concevoir et gérer la performance dans l'Administration, Québec: Presses de l'Université du Québec, 440 p.

3 Assemblée nationale du Québec, Loi sur l'administration publique, L. R. Q. Chapitre A-6.01.

4 OCDE (2005). Moderniser l'État, La route à suivre. Éditions OCDE, 265 p. (p. 83 et pp. 182-3).

5 Kernaghan, K., B. Marson et B. Sandford (2001). L'administration publique de l'avenir. Toronto : Institut d'administration publique du Canada, $390 \mathrm{p}$.

6 Bouckaert, G. (2005). «Un nouvel examen de la mesure de la performance dans le secteur public », Télescope, vol. $12, \mathrm{n}^{\mathrm{o}} 3$.

7 Good, D. A. (2003). The Politics of Public Management, Toronto : University of Toronto Press.

8 Paquet, G. (2009). « Quantophrenia », Optimum, 39;1.

9 Savoie, D. J. (2008). Court Government and the Collapse of Accountability in Canada and the United Kingdom. Toronto : University of Toronto Press, 441 p.

${ }^{10}$ Radin, B. A. (2006). Challenging the Performance Movement, Accountability, Complexity and Democratic Values, Washington, D. C. : Georgetown University Press, $260 \mathrm{p}$.

${ }^{11}$ Suleiman, E. (2005). Le démantèlement de l'État démocratique, Éditions du Seuil, 401 p. (pp. 33-34).

${ }^{12}$ Wilson, J. Q. (1989). Bureaucracy, What Government Agencies Do and Why They Do It, Basic Books, 433 p. (pp. 375-6). 


\section{Notes}

${ }^{a}$ Le lecteur qui désire approfondir les particularités de cette approche dans le cadre plus large des courants théoriques ayant marqué le développement de l'administration publique comme discipline pourra consulter Frederickson, H. G et K. B. Smith. (2003). The Public Administration Theory Primer, Westview Press.

${ }^{\mathrm{b}}$ L'analogie entre une discipline sportive et la performance d'une organisation publique n'est pas sans intérêt. Les finalités varieront selon la discipline (courir le 100 mètres et le lancer du javelot selon des standards olympiques) ainsi que les moyens les plus appropriés pour les réaliser (entraînements et diètes adaptés). Les missions (finalités) des organisations publiques peuvent elles aussi être très différentes et faire appel à des moyens pour les poursuivre.

${ }^{c}$ La «gestion par objectifs » et la «gestion axée sur les résultats » se distinguent pour l'essentiel par une différence de perspective temporelle, l'objectif précédant l'action alors que le résultat en découle. On peut définir des objectifs en négligeant de vérifier si les résultats obtenus leur sont conformes comme on peut produire et observer des résultats «non recherchés » c'est à dire qui ne découleraient pas d'objectifs prédéterminés. La gestion axée sur les résultats promeut la production de résultats à partir d'objectifs convenus.

${ }^{\mathrm{d}} \mathrm{Ce}$ que nous appelons ici la «modernisation des administrations » ne débute pas dans les années 80 avec le mouvement dit du «nouveau management public», évoqué en début d'article. Bien qu'il ne soit pas question ici de préciser la portée du terme, on conviendra, par exemple, que la modernisation de l'administration publique québécoise remonte bien avant cette date soit au début des années 60 .

e Tirées de l'ouvrage de référence et complétées par l'auteur de cet article.

${ }^{\mathrm{f}}$ On notera que les auteurs du rapport de l'OCDE utilisent le terme «performances » au pluriel en mettant l'accent sur une des deux dimensions du concept évoquées au début de l'article, celle des résultats ultimes.

${ }^{g} \mathrm{Au}$ Canada, par exemple, les organisations publiques fédérales doivent assurer une accessibilité des services dans les deux langues officielles. Elles doivent aussi chercher à accueillir dans leurs rangs des personnes issues de milieux socio-économiques variés. Enfin, elles doivent se plier à des obligations en matière d'accès à l'information. Ce sont là autant d'exigences non imposées aux entreprises ou organisations du secteur privé.

h À la mi-janvier 2000, le contrôleur interne du Ministère des ressources humaines du Canada rend public son rapport qui vaudra à ce ministère de vivre une crise qui se terminera 10 mois plus tard avec le dépôt du rapport du Vérificateur général du Canada. L'auteur qui fût sousministre adjoint du Ministère jusqu'en 2000 décrit les événements à partir de différentes perspectives et montre comment une réduction importante du personnel, des changements importants dans les programmes concernés, la volonté d'améliorer les services offerts, l'élimination de certaines procédures administratives et le rôle accru des parlementaires ont contribué à l'affaiblissement de la gestion et du contrôle des dépenses (p. 9). Une formidable étude de cas qui montre comment le «nouveau management public » peut produire des résultats discutables. 


\section{Les études de cycles supérieurs à I'UQAC}

AFTS ET LETTRES * Programme wurt en didectique du fançais lengue matemelle * Meitrise en

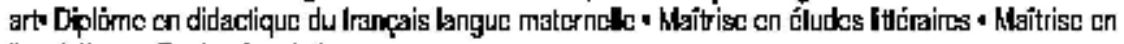
linguistique - Doctoral en le.tres

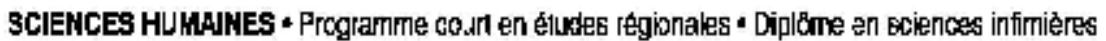
- Meîtrise en médecine expéfimentale génétique des populations - Maîtrise en études et intervenlions

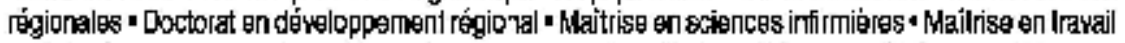
Bociel * Programme court en ethique * Programme court en ét.des religieusees * Dipkime en éthique de

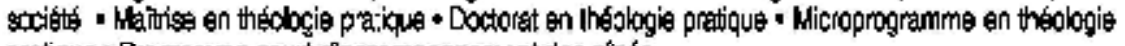
pratique - Programme court dfacompagnement des aíness

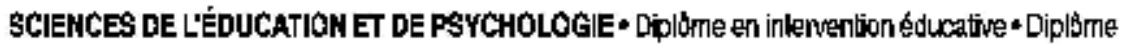

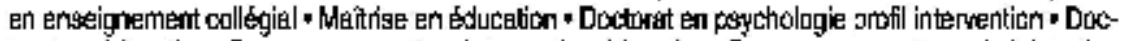
tora: en éducation * Prograrrre courl en injensention éducatił * Programme court en administration scolalie • Diplorre en administratlon scolalre

SCIENCES ĖCONOUIquES ET ADIINISTRATIVES - Programme court en gesilon de projel

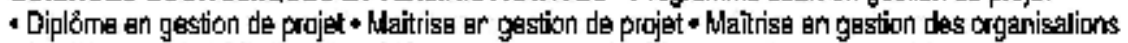
- Maltrise en administralicn des affaires pour cadres - Diphöme en sciences comptables

SCIENCES APPLIQUÉES - Mailtrise en ingénierie • Dociorat en ingénierie • Diplöme en sciences de la Terre $\cdot$ Maitrise en sciences de la Terre $•$ Doctorat en resscurces minérales

SCIENCES FONDAMENTALES - Maîtrise en ressources renowvelables - Doctorat en sciences de lenvironnement • Diplöme en éco-conseil

INFORMATIQUE ET MATHÉMATIQUES

- Diplôme en informatque appliquée • Maítrise en informatique

2,5 millions \$

pour les étudiantes et les étudiants

en support et formation à la recherche Consultez nos programmes

www uqac.ca

UQAC

UNIVERSITÉ DU QUÉBEC À CHICOUTIMI

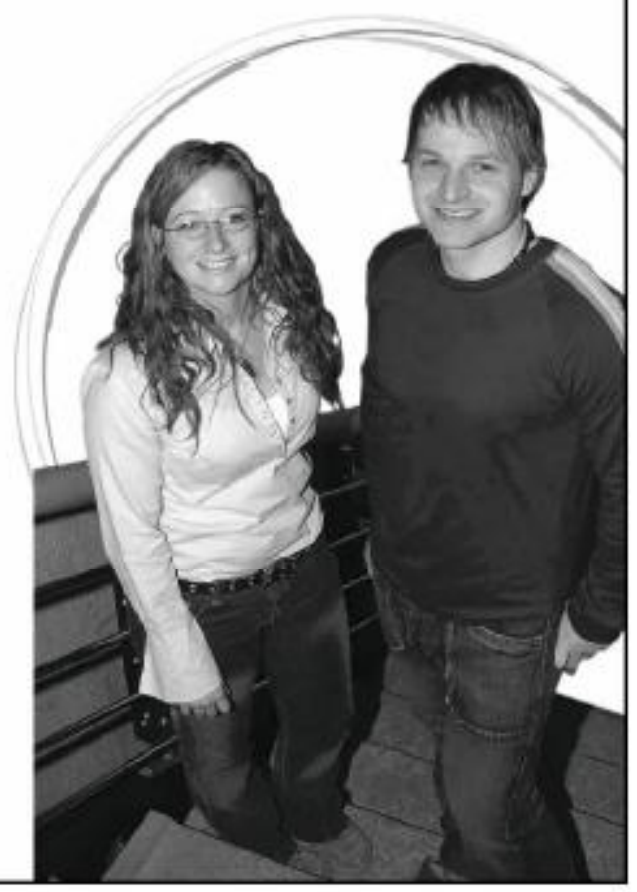

